\title{
Adaptive Consensus via Dynamic Feedback Control for Lipschitz Nonlinear Multi-Agent Systems
}

Lin Li

Department of Control Science and Engineering, University of Shanghai for Science and Technology, 516 JunGong Road, Shanghai, 200093, P.R.China

Heyang Wang

Department of Control Science and Engineering, University of Shanghai for Science and Technology, 516 JunGong Road, Shanghai, 200093, P.R.China

E-mail: lilin0211@163.com,wangheyang2015@163.com

www.usst.edu.cn

\begin{abstract}
This paper deals with the consensus problem for multi-agent systems with fixed topologies. The agents are described by Lipschitz nonlinear systems. Only output information of each agent can be obtained from its neighbor agents. A distributed adaptive consensus algorithm via dynamic output feedback is proposed, in which the coupling weights between adjacent agents are time-varying and satisfy some designed adaptive laws. Provided examples are included to demonstrate the effectiveness of the proposed consensus algorithm.
\end{abstract}

Keywords: consensus, multi-agent systems, adaptive protocol, dynamic output feedback, Lipschitz nonlinear

\section{Introduction}

With the rapid development of science and technology, the distributed coordinated control problem for multiagent systems (MASs) has received increasing attention. Consensus is an important and fundamental issue for coordinated control of the MASs, and has received more and more attention due to its broader range applications, such as flocking control, tracking control, unmanned aerial vehicle formation, underwater robot coordinated search and rescue, etc. Over the past decade, research on consensus problem has achieved numerous meaningful results. ${ }^{1-6}$ Above the existing works that about consensus control for MASs are based on static state-feedback under a restrictive assumption that all the agents' states can be measured, while the global information, for example the eigenvalue of the Laplace matrix, is used. But we know that in many real applications, the full states of the agents cannot be got, and the global information of the MASs may be unknown. Recently, an adaptive protocol was proposed to solve consensus for general linear MASs, ${ }^{7}$ where the states of agents were supposed to be known. The observer-based protocol was investigated. ${ }^{8}$

In this paper, the consensus problem of MASs with Lipschitz nonlinear dynamics under a fixed undirected connected topology is in focus. A distributed output feedback protocol is proposed, in which the output errors of the agents and the controller states' errors are 
used to be the controller input. And in this designed protocol, the coupling weights between agents satisfy some designed adaptive law, in this way, the global information are avoided. Sufficient condition for the existence of this protocol is derived. Finally, numerical simulation shows the effectiveness of our obtained method.

\section{Problem Formulation}

The communication topology among agents is represented by a weighted connected undirected graph $\mathrm{G}=(\mathrm{V}, \mathrm{E}, \mathrm{A})$. Hereinto, $\mathrm{V}$ represents the set of nodes with $\mathrm{V}=\left\{v_{1}, v_{2}, \cdots v_{N}\right\}, \mathrm{E}$ is the set of undirected edges, and $\mathrm{A}=\left[a_{i j}\right]$ is the weighted adjacency matrix with $a_{i i}=0, a_{i j}>0$. An undirected edge $a_{i j}$ is denoted by the pair of nodes $\left(v_{i}, v_{j}\right) \in \mathrm{E}$. The in-degree of nodes $v_{i}$ is defined by $\mathrm{D}_{\text {in }}(i)=\sum_{j} a_{i j}$. Laplace matrix is defined as $L=\left[l_{i j}\right]=\Delta-A, \Delta=\operatorname{diag}\left\{\mathrm{D}_{\text {in }}(1), \cdots \mathrm{D}_{\text {in }}(N)\right\}$.

Consider a multi-agent system consisting of $N$ agents, and the dynamic of the $i$ th agent is

$\dot{x}_{i}(t)=A x_{i}(t)+f\left(x_{i}\right)+B u_{i}(t)$

$y_{i}(t)=C x_{i}(t) \quad i=1,2, \cdots N$

where $x_{i}(t)$ is the state, $y_{i}(t)$ is the measured output, and $u_{i}(t)$ is the control input of the $i$ th agent. $A, B$ and $C$ are known real matrices. The nonlinear function $f\left(x_{i}\right)$ satisfies the following Lipschitz condition:

$\|f(x)-f(y)\| \leq \mu\|x-y\|$

where $\mu>0$ is the known Lipschitz constant.

For system (1), since some states cannot be measured in many cases, consider the following distributed consensus protocol

$$
\begin{aligned}
& \dot{x}_{o i}(t)=A_{o} x_{o i}(t)+f\left(x_{o i}\right)+B_{o} \sum_{j} c_{i j}(t) a_{i j} \\
& {\left[y_{i}(t)-y_{j}(t)+C\left(x_{o i}(t)-x_{o j}(t)\right)\right]} \\
& u_{i}(t)=C_{o} x_{o i}(t) \quad i=1,2, \cdots N
\end{aligned}
$$

where $x_{o i}(t)$ is the state, $A_{o}, B_{o}$ and $C_{o}$ are the gain matrices to be determined, $c_{i j}(t)$ is the time-varying coupling weight of agent $i$ and agent $j$ satisfying $c_{i j}(0)=c_{j i}(0)$.

The objective of this paper is to design a distributed consensus protocol (3) with an adaptive law about the $c_{i j}(t)$ for the group of agents such that the states of system (1) achieve consensus asymptotically. That is, for any initial states $x_{i}(0)$, the following is satisfied $\lim _{t \rightarrow \infty}\left\|x_{i}(t)-x_{j}(t)\right\|=0, i, j=1,2, \ldots, N$
Denote
$e_{i}(t)=x_{i}(t)-\frac{1}{N} \sum_{j=1}^{N} x_{j}(t)$

and

$e_{i}(t)=x_{i}(t)-\frac{1}{N} \sum_{j=1}^{N} x_{j}(t)$.

Then, it follows form (1) and (3) that

$$
\begin{gathered}
\dot{e}_{i}(t)=A e_{i}(t)+f\left(x_{i}\right)-\frac{1}{N} \sum_{j=1}^{N} f\left(x_{j}\right)+B C_{o} e_{o i} \\
\dot{e}_{o i}(t)=A_{o} e_{o i}(t)+f\left(x_{o i}\right)-\frac{1}{N} \sum_{j=1}^{N} f\left(x_{o j}\right)+B_{o} C \sum_{j} c_{i j}(t) a_{i j}\left(e_{i}(t)-e_{j}(t)\right) \\
+B_{o} C \sum_{j} c_{i j}(t) a_{i j}\left(e_{o i}(t)-e_{o j}(t)\right)
\end{gathered}
$$

Choose the adaptive law described as follows

$\dot{c}_{i j}(t)=-a_{i j}\left[\left(e_{o i}(t)-e_{o j}(t)\right)^{T} Q B_{o} C\left(e_{i}(t)-e_{j}(t)\right)\right.$

$$
\left.+\left(e_{o i}(t)-e_{o j}(t)\right)^{T} Q B_{o} C\left(e_{o i}(t)-e_{o j}(t)\right)\right], i, j=1,2, \cdots N
$$

If $\lim _{t \rightarrow \infty}\left\|e_{i}(t)-e_{j}(t)\right\|=0$ and $\lim _{t \rightarrow \infty}\left\|e_{o i}(t)-e_{o j}(t)\right\|=0$. We can get that $\lim _{t \rightarrow \infty}\left\|x_{i}(t)-x_{j}(t)\right\|=0$. Then, the consensus problem of system (1) is equal to the asymptotical stability of the error system (4). In the following, we will focus on the asymptotical stability of system (4).

\section{Main Result}

Theorem 1. Given positive scalar $\mu$, if there exist a constant $\beta>0$, positive definite matrices $P, R$, and three matrices $V_{i}(i=1,2,3)$ such that the following LMI holds

$$
\left[\begin{array}{ccccccc}
(1,1) & B V_{3} & V_{2} & P & 0 & 0 & 0 \\
* & (2,2) & V_{2} & 0 & P & \mu R & C^{T} \\
* & * & -I & 0 & 0 & 0 & 0 \\
* & * & * & -I & 0 & 0 & 0 \\
* & * & * & * & -I & 0 & 0 \\
* & * & * & * & * & -I & 0 \\
* & * & * & * & * & * & -\beta I
\end{array}\right]<0
$$

where $(1,1)=A P+P A^{T}+\mu^{2} I,(2,2)=V_{1}+V_{1}^{T}$. The signal * denotes the symmetry part of a symmetry matrix. Then, under the protocol (3) with the adaptive law (5), the consensus of multi-agent system (1) can be solved. And the gain matrices of this protocol can be taken as: $A_{o}=R^{-1} V_{1} P^{-1}, B_{o}=R^{-1} V_{2}, C_{o}=V_{3} P^{-1}$.

Proof: For system (4), consider the following Lyapunov-Krasovskii functional candidate 
$V(t)=\sum_{i=1}^{N} e_{i}^{T}(t) P^{-1} e_{i}(t)+\sum_{i=1}^{N} e_{o i}^{T}(t) Q e_{o i}(t)+\sum_{i=1}^{N} \sum_{j=1}^{N} \frac{\left(c_{i j}(t)+\alpha\right)^{2}}{2}$

where $\alpha$ is some appropriate constant. Taking the time derivative of Lyapunov functional $V(t)$ along the trajectory of system (4), we get

$$
\begin{aligned}
\dot{V}(t)= & 2 \sum_{i=1}^{N} e_{i}^{T}(t) P^{-1} \dot{e}_{i}(t)+2 \sum_{i=1}^{N} e_{o i}^{T}(t) Q \dot{e}_{o i}(t) \\
& +\sum_{i=1}^{N} \sum_{j=1}^{N}\left(c_{i j}(t)+\alpha\right) \dot{c}_{i j}(t) .
\end{aligned}
$$

From (4) and (5), we have

$$
\begin{aligned}
& \dot{V}(t)=2 \sum_{i=1}^{N} e_{i}^{T}(t) P^{-1}\left[A e_{i}(t)+f\left(x_{i}\right)-\frac{1}{N} \sum_{j=1}^{N} f\left(x_{j}\right)\right. \\
& \left.+B C_{o} e_{o i}\right]+2 \sum_{i=1}^{N} e_{o i}^{T}(t) Q\left[A_{o} e_{o i}(t)+f\left(x_{o i}\right)-\frac{1}{N} \sum_{j=1}^{N} f\left(x_{o j}\right)\right. \\
& \left.+\alpha B_{o} C \sum_{j} c_{i j}(t) a_{i j}\left(e_{i}(t)-e_{j}(t)+e_{o i}(t)-e_{o j}(t)\right)\right]
\end{aligned}
$$

Since $c_{i j}(t)=c_{j i}(t)$, we get

$$
\begin{aligned}
& 2 \sum_{i=1}^{N} e_{o i}^{T}(t) Q B_{o} C \sum_{j} c_{i j}(t) a_{i j}\left(e_{o i}(t)-e_{o j}(t)\right) \\
& =\sum_{i=1}^{N} \sum_{j} c_{i j}(t) a_{i j}\left(e_{o i}(t)-e_{o j}(t)\right)^{T} Q B_{o} C\left(e_{o i}(t)-e_{o j}(t)\right)
\end{aligned}
$$

Denote $\bar{x}=\frac{1}{N} \sum_{j=1}^{N} x_{i}(t), \bar{x}_{o}=\frac{1}{N} \sum_{j=1}^{N} x_{o i}(t)$, we have

$$
\begin{aligned}
& f\left(x_{i}\right)-\frac{1}{N} \sum_{j=1}^{N} f\left(x_{j}\right)=f\left(x_{i}\right)-f(\bar{x})+f(\bar{x})-\frac{1}{N} \sum_{j=1}^{N} f\left(x_{j}\right) \\
& f\left(x_{o i}\right)-\frac{1}{N} \sum_{j=1}^{N} f\left(x_{o j}\right)=f\left(x_{o i}\right)-f\left(\bar{x}_{o}\right)+f\left(\bar{x}_{o}\right)-\frac{1}{N} \sum_{j=1}^{N} f\left(x_{o j}\right)
\end{aligned}
$$

In addition, because $\sum_{i=1}^{N} e_{i}(t)=0, \sum_{i=1}^{N} e_{o i}(t)=0$, we get

$$
\begin{aligned}
& 2 \sum_{i=1}^{N} e_{i}^{T}(t) P^{-1}\left[f(\bar{x})-\frac{1}{N} \sum_{j=1}^{N} f\left(x_{j}\right)\right]=0 \\
& 2 \sum_{i=1}^{N} e_{o i}^{T}(t) Q\left[f\left(\bar{x}_{o}\right)-\frac{1}{N} \sum_{j=1}^{N} f\left(x_{o j}\right)\right]=0
\end{aligned}
$$

Then, it follows from the Lipschitz condition (2) that

$$
\begin{aligned}
& 2 e_{i}^{T}(t) P^{-1}\left[f\left(x_{i}\right)-\frac{1}{N} \sum_{j=1}^{N} f\left(x_{j}\right)\right] \leq e_{i}^{T}(t)\left(\mu^{2}\left(P^{-1}\right)^{2}+I\right) e_{i}(t) \\
& 2 e_{o i}^{T}(t) Q\left[f\left(x_{o i}\right)-\frac{1}{N} \sum_{j=1}^{N} f\left(x_{o j}\right)\right] \leq e_{o i}^{T}(t)\left(\mu^{2} Q^{2}+I\right) e_{o i}(t)
\end{aligned}
$$

Let

$$
\begin{aligned}
& \tilde{e}=\left[e_{1}^{T}(t), e_{2}^{T}(t), \cdots, e_{N}^{T}(t)\right]^{T} \\
& \tilde{e}_{o}=\left[e_{o 1}^{T}(t), e_{o 2}^{T}(t), \cdots, e_{o N}^{T}(t)\right]^{T} \\
& \xi=\left[\tilde{e}^{T}, \tilde{e}_{o}^{T}\right]^{T}
\end{aligned}
$$

Combing (5), (9) and (10) in to (8) yields
$\dot{V}(t) \leq \xi^{T} \Phi \xi$

where

$$
\begin{aligned}
& \Phi=\left[\begin{array}{cc}
\Phi_{11} & \Phi_{12} \\
* & \Phi_{22}
\end{array}\right], \\
& \Phi_{11}=I_{N} \otimes\left(P^{-1} A+A^{T} P^{-1}+\mu^{2}\left(P^{-1}\right)^{2}+I\right) \\
& \Phi_{12}=I_{N} \otimes\left(P^{-1} B C_{o}\right)+\alpha L \otimes Q B_{o} C \\
& \Phi_{22}=I_{N} \otimes\left(Q A_{o}+A_{o}^{T} Q+\mu^{2} Q^{2}+I\right)+2 \alpha L \otimes Q B_{o} C
\end{aligned}
$$

The graph $\mathrm{G}$ is connected, zero is a simple eigenvalue of Laplace matrix ${ }_{L}$, and all the other eigenvalues are positive. Then, there exists a unitary matrix $U \in \mathrm{R}^{N \times N}$ such that $U^{T} L U=\bar{L}=\operatorname{diag}\left\{0, \lambda_{1}, \lambda_{2}, \cdots \lambda_{N}\right\}$, where $\lambda_{i}(i=2,3, \cdots, N)$ are positive eigenvalues of $L_{L}$. Let $\bar{\xi}=\operatorname{diag}\left\{U^{T} \otimes I_{N}, U^{T} \otimes I_{N}\right\} \xi$, we have $\dot{V}(t) \leq \bar{\xi}^{T} \bar{\Phi} \bar{\xi}$

If the inequality (6) is satisfied, choose $\alpha$ such that $\alpha^{2} \leq \frac{\beta}{\lambda_{N}^{2}}$, and let $R=P Q$, it is easy to get that $\dot{V}(t)<0$. Hence, $V(t)$ is bounded. By using the LaSalle's Invariance Principle, the error system (4) is asymptotically stable. Then, the consensus problem of system (1) is solved. This completes the proof.

\section{Simulation Example}

A simulation example is illustrated to show the effectiveness of our results. Consider an undirected graph $\mathrm{G}$, the communication topology is the same as Fig. $1^{8}$, in which there are eight agents. The elements of adjacency matrix A satisfy $a_{i j}=a_{j i}=1$ with $\left(v_{i}, v_{j}\right) \in \mathrm{E}$. Consider system (1) with

$$
\begin{aligned}
& A=\left[\begin{array}{cc}
-0.9 & 0 \\
0 & -2
\end{array}\right], B=\left[\begin{array}{l}
0.1 \\
0.2
\end{array}\right], C=\left[\begin{array}{cc}
0.5 & 0.2 \\
0 & 0.3
\end{array}\right], \\
& f\left(x_{i}\right)=\left[\begin{array}{ll}
0 & -0.1 \sin \left(x_{i 2}\right)
\end{array}\right]^{T}
\end{aligned}
$$

It is easy to see that the nonlinear dynamic $f\left(x_{i}\right)$ satisfies the condition (2) with $\mu=0.1$. Suppose the initial states of system (1) are $x_{1}(0)=\left[\begin{array}{ll}5 & 1\end{array}\right]^{T}$, $x_{2}(0)=\left[\begin{array}{ll}0 & -1\end{array}\right]^{T}, \quad x_{3}(0)=\left[\begin{array}{ll}9 & 1\end{array}\right]^{T}, \quad x_{4}(0)=\left[\begin{array}{ll}-5 & 1\end{array}\right]^{T}$, $x_{6}(0)=\left[\begin{array}{ll}2 & 0\end{array}\right]^{T}, x_{7}(0)=\left[\begin{array}{ll}-1.8 & -1\end{array}\right]^{T}, x_{8}(0)=\left[\begin{array}{ll}-6 & 2\end{array}\right]^{T}$. By solving the inequality (6), we can obtain the dynamic output feedback gain matrices are

$$
\begin{aligned}
& A_{o}=\operatorname{diag}(-0.2,-0.2), B_{o}=\left[\begin{array}{cc}
-0.2040 & 0 \\
0 & -0.2040
\end{array}\right] \\
& C_{o}=\left[\begin{array}{ll}
1.3945 & 1.9870
\end{array}\right]
\end{aligned}
$$



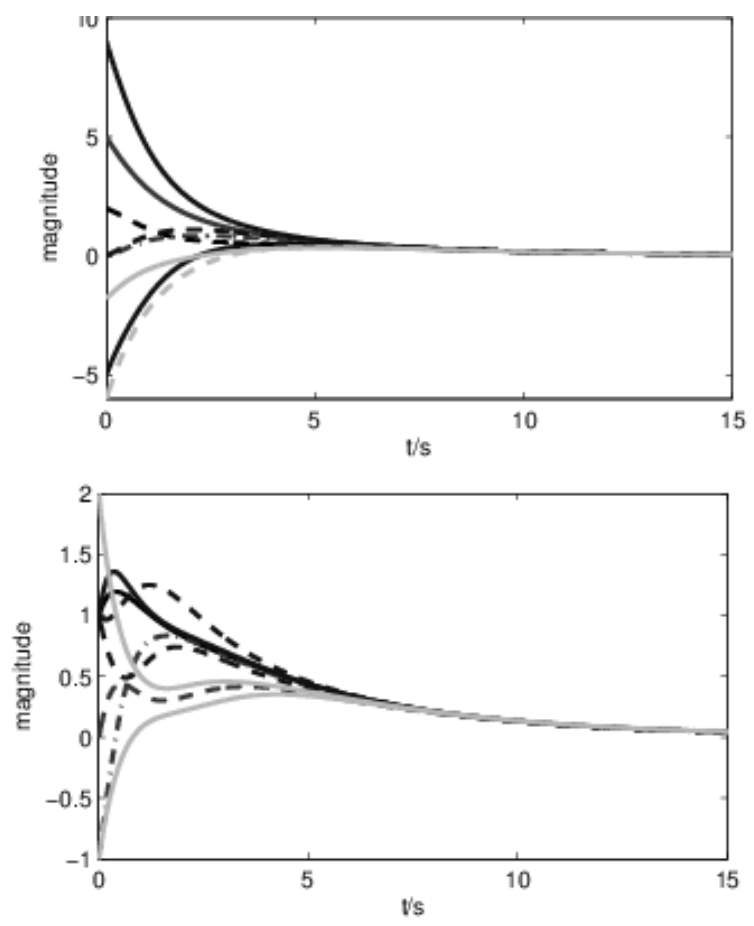

Fig. 1. The states of multi-agent system (1)

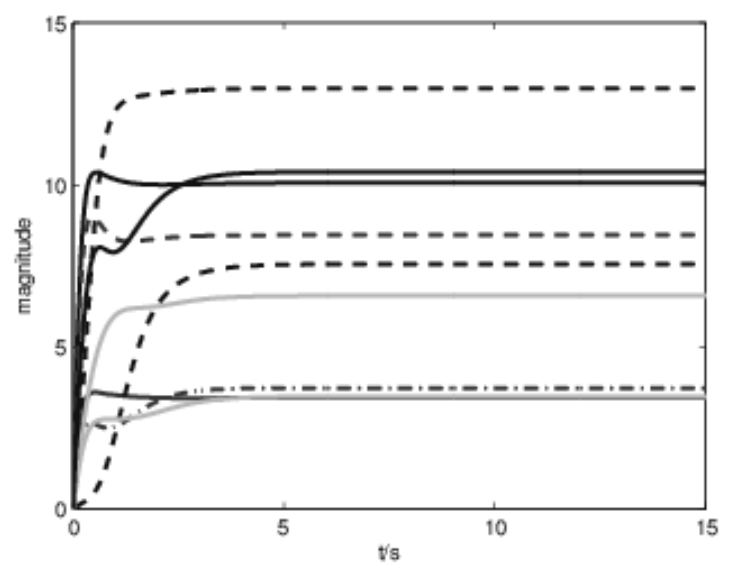

Fig. 2. The coupling weights $c_{i j}(t)$

The state trajectories of nonlinear multi-agent system (1) are shown in Fig. 1. From Fig.1, we can see that the consensus control problem of system (1) under the distributed adaptive protocol (3) can be asymptotically solved. Curves of coupling weights are given in Fig.2.

\section{Conclusion}

The state consensus problem for multi agent systems with Lipschitz nonlinear dynamics has been investigated. Under that consideration of the unmeasured agent state, a distributed dynamic output feedback protocol with time varying coupling weights was designed. The coupling weights satisfy the designed adaptive law. A simulation example shows that the consensus problem under the designed distributed adaptive protocol can be asymptotically solved.

\section{Acknowledgements}

This paper is supported by the National Natural Science Foundation (61203143), and the Hujiang Foundation of China (C14002).

\section{References}

1. T. Li, M.Y. Fu, L.H. Xie, J.F. Zhang, Distributed consensus with limited communication data rate, IEEE Transaction on Automatic Control 56(2) ( 2011) 279291.

2. Z.W. Liu, et al, Consensus of multi-agent networks with a periodic sampled communication via impulsive algorithms using position-only measurement, IEEE Transaction on Automatic Control 57(10) ( 2012 ) 26392643.

3. M. Ji and M. Egerstedt, Distributed coordination control of multi-agent systems while preserving connectedness, IEEE Transactions on Robotics 23(4) (2007) 693-703.

4. R. Olfati-Saber and R.M. Murry, Consensus problems in networks of agents with switching topology and timedelays, IEEE Transaction on Automatic Control 49(9) (2004) 1520-1533.

5. W. Ren and E.M. Atkins, Distributed multi-vehicle coordinated control via local information exchange, International Journal of Robust and Nonlinear Control 17(1011) (2007) 1002-1033.

6. P. Lin, Y. Jia, Distributed robust $\mathrm{H}_{\infty}$ consensus control in directed networks of agents with time-delay, Systems \& Control Letters 57(8) (2008) 643-653.

7. J. Hu, et al., Consensus of nonlinear multi-agent systems with observer-based protocols, Systems \& Control Letters 72(10) (2014) 71-79.

8. Z. Li, et al., Consensus of multi-agent systems with general linear and Lipschitz nonlinear dynamics using distributed adaptive protocols, IEEE Transactions on Automatic Control 58(7) (2013) 1786-1791. 